\title{
SolpeX: the soft X-ray flare polarimeter-spectrometer for the ISS
}

\author{
Janusz Sylwester ${ }^{1}$, Stefan Płocieniak ${ }^{1}$, Jarosław Ba̧kała ${ }^{1}$, \\ Żaneta Szaforz ${ }^{1}$, Marek Stȩślicki ${ }^{1}$, Daniel Ścisłowski ${ }^{1}$, \\ Mirosław Kowaliński ${ }^{1}$, Piotr Podgórski ${ }^{1}$, Jose Hernandez ${ }^{1}$ and \\ Sergey Shestov ${ }^{2}$ \\ ${ }^{1}$ Space Research Centre, Polish Academy of Sciences, \\ 51-622 Wrocław, ul. Kopernika 11, Poland \\ email: js@cbk.pan.wroc.pl \\ ${ }^{2}$ P.N. Lebedev Physical Institute, Russian Academy of Sciences, \\ 53 Leninskij Prospekt, 119991, Moscow, Russia \\ email: sshestov@sci.lebedev.ru
}

\begin{abstract}
We present the innovative soft X-ray spectro-polarimeter, SolpeX. This instrument consists of three functionally independent blocks. They are to be included into the Russian instrument KORTES, to be mounted onboard the ISS. The three SolpeX units are: a simple pin-hole X-ray spectral imager, a polarimeter, and a fast-rotating drum multiple-flat-crystal Bragg spectrometer. Such a combination of measuring blocks will offer a new opportunity to reliably measure possible X-ray polarization and spectra of solar flares, in particular during the impulsive phase. Polarized Bremsstrahlung and line emission due to the presence of directed particle beams will be detected, and measurements of the velocities of evaporated hot plasma will be made. In this paper we discuss the details of the construction of the SolpeX units. The delivery of KORTES with SolpeX to the ISS is expected to happen in 2017/2018.
\end{abstract}

Keywords. solar flares, soft X-rays, polarimetry, spectroscopy, ISS

\section{Introduction}

Studying the nature of flare X-ray sources is crucial for understanding the conversion of magnetic energy coming from reconnecting fields. A part of the non-potential magnetic energy is used to accelerate electrons and protons, forming highly anisotropic beams that propagate along the field lines towards denser atmospheric regions. These beams, while interacting with the ambient dense plasma, release a small part of energy in the form of non-thermal Bremsstrahlung, which is expected to be polarized due to the anisotropy of the interactions. With SolpeX, we intend to detect this polarization for the first time in soft X-ray line and continuum emission around $\sim 3 \mathrm{keV}$; additionally, we intend to constrain the properties of electron beams by determining the orientation of the magnetic field in the interaction region (Emslie et al. 2008). Models of interaction predict that the harder X-ray emission, consisting solely of continuum emission, should be highly polarized, with a polarization degree as high as $40 \%$ at $20 \mathrm{keV}$ (Zharkova et al. 2010). The soft X-ray emission (at energies $<10 \mathrm{keV}$ ), which contains emission lines seen atop the continuum, is also expected to be polarized (Elwert \& Haug 1970). The $\mathrm{X}$-rays from anisotropic interactions are expected to dominate during the early impulsive phase of flares (with durations of seconds to minutes). Although at a lower level, the other processes contributing to polarization in the later flare phases are caused by the anisotropy of the electron distribution function within thermalized sources (Emslie 

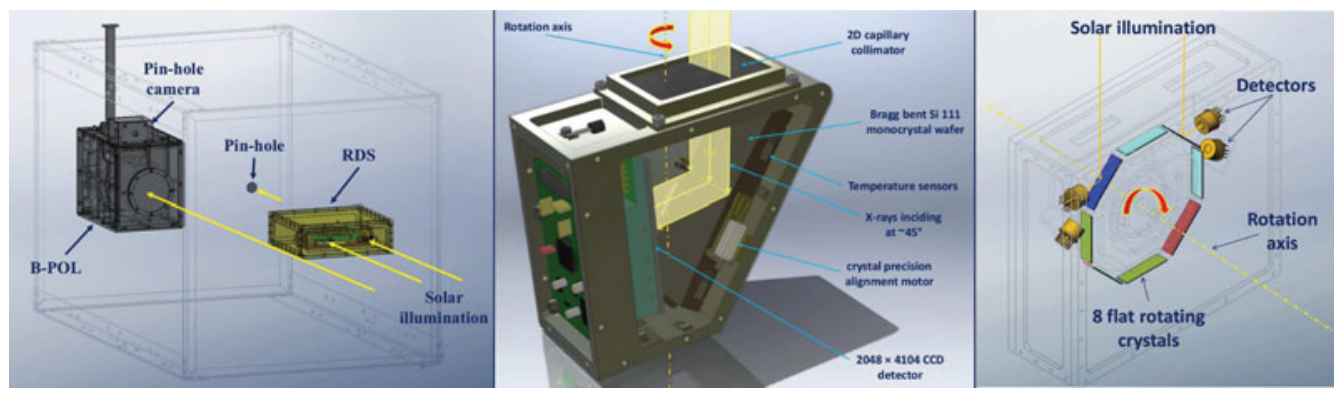

Figure 1. Left: Placement of the pin-hole, B-POL and the RDS blocks within the Russian-built KORTES instrument; Middle: the polarimeter of B-POL; The rotation axis (1 rotation/second) is to be pointed towards the flare. The $2 \mathrm{D}$ collimator $(2 \operatorname{arcmin} \times 2 \operatorname{arcmin})$ "isolates" the source from the rest of X-ray disk emission; Right: The RDS main components. Eight crystals (two of them identical) are fixed to the rotating (10 rps) drum. Bragg reflected spectra are recorded by four SDD detectors.

\& Brown 1980) and/or backscattering of the X-rays from lower-lying coronal sources of the solar atmosphere (Jeffrey \& Kontar 2011).

The importance of measuring the polarization of X-ray flare emission was realized at the very beginning of the space era. The first attempts at detecting X-ray polarization from solar flares were made using polarimeters on-board Soviet Intercosmos satellites. The most recent determinations of solar flare X-ray polarization at the harder energy range were obtained from the analysis of data collected by RHESSI and the SPR-N polarimeter on-board the CORONAS-F satellite.

Using RHESSI data McConnell et al. (2003) found a polarization level of $\sim 18 \%$ in the 20-40 keV energy range. The source was an X-class solar flare that took place on July 23, 2002 at 00:35 UT (SOL2002-07-23T00:35). Polarization levels of 21\% $\pm 9 \%$ and $11 \% \pm 5 \%$ for this and another X-class flare (SOL2003-10-28T11:06), respectively, were reported by Boggs et al. (2006) for a much higher energy range $(0.2-1 \mathrm{MeV})$. SuarezGarcia et al. (2006) found, for six X-class flares and one M-class flare, values of the degree of polarization between $2 \%$ and $54 \%$, for an energy range between 100 and $350 \mathrm{keV}$.

Using the SPR-N polarimeter on-board the CORONAS-F satellite Zhitnik et al. (2006) found, among 90 analyzed flares, one event (X10 class flare SOL2003-10-29T20:37) which showed a polarization degree exceeding $70 \%$ at energies between $40-100 \mathrm{keV}$, and of about $50 \%$ at lower energies $(20-40 \mathrm{keV})$. For 25 of the events, the upper limits for the polarization were estimated to be between $8 \%$ and $40 \%$.

All these determinations of X-ray polarization for flares were obtained using a Compton scattering technique. For polarized radiation, the azimuthal distribution of the scattered photons is no longer isotropic, but it is related to the polarization vector of the incident photons. The energy resolution of the Compton polarimeters was rather coarse. Also, a low signal-to-noise ratio did not allow for high temporal resolution. Therefore, we decided to try a different detection technique with the aim of studying the polarization in a softer X-ray band, i.e. the dependence of the total coefficient of Bragg reflection (close to the Brewster angle), on the orientation of the polarization vector relative to the crystal plane (cf. Fig. 1).

Below, we describe in some detail the construction of the SolpeX blocks, designed to observe solar soft X-ray emission. The instrument consists of three measuring units placed within the KORTES instrument, which will be mounted on top of the new Russian science module, "Nauka", and will be delivered and attached to the ISS soon.

The SolpeX individual measuring units are: 

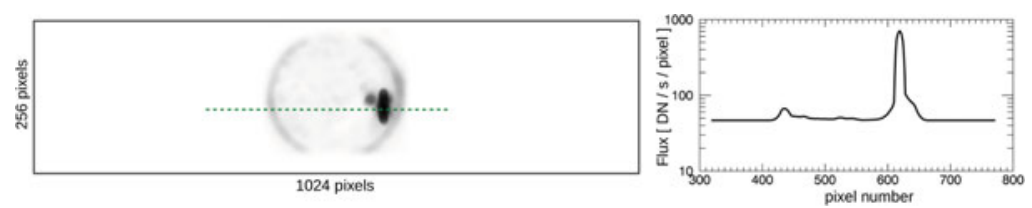

Figure 2. Simulated X-ray image of the Sun, with a C5 class flare in progress, projected on the pinhole CCD camera (left). The signal profile "observed" along the dashed line cut is shown to the right.

- PHI - a simple Pin-Hole soft X-ray Imager-spectrophotometer with moderate spatial $(\sim 20$ arcsec $)$ and spectral $(0.5 \mathrm{keV})$ resolutions, and high cadence $(0.1 \mathrm{~s})$.

- B-POL - the $3.9 \AA-4.6 \AA$ Bragg POLarimeter with a low (1\%-2\%) linear polarization detection threshold.

- RDS - a fast Rotating Drum X-ray Spectrometer with high time resolution $(0.1 s)$

In Fig. 1, we show the location of the units within the KORTES instrument. The RDS unit is placed close to the radiator, which is common to the entire instrument. The BPOL polarimeter is placed towards the rear of the instrument. This location is imposed by functionality, as the polarimeter's CCD detector needs to be cooled down to below $-20^{\circ} \mathrm{C}$ using a rotating radiator fixed to the rotating axis of the polarimeter (this also acts as a heat sink). Attached to the side of the polarimeter, there is another smaller CCD onto which the X-ray pin-hole image of the Sun is projected. The pin-hole itself is mounted on the front-side of the instrument. KORTES is to be placed on the solar pointing platform attached to the ISS. This platform is oriented towards the center of the solar disk. The illumination conditions at the location of KORTES are not ideal for carrying out solar observations, since the complicated mechanical structure of the ISS causes substantial vignetting. Only 10-12 min of uninterrupted illumination is available for every ISS orbit, so catching the impulsive phase of a flare will need some luck. Estimates indicate that in 2017 , around the time of solar minimum, we can expect to observe the impulsive phases of $\sim 40$ C-class flares, $5 \mathrm{M}$-class flares and $0.5 \mathrm{X}$-class flare during one year of instrument operation in orbit. The KORTES instrument will be delivered to ISS by a cargo ship and then fixed to the Nauka module by cosmonauts. After the mission is completed (or in case of failure), the instrument will return to the ground for post-flight checks. The data from the instrument will be stored on the recorder inside ISS, so practically unlimited storage is available on hard disk. A selected subset of the data will be telemetered down to Earth for prompt analysis that will allow us to implement software corrections and upgrades. The instrument can be operated round the clock. During the night-portions of the orbit it will be run for calibration purposes or for measurements of non-solar astrophysical sources. Below we describe each block of SolpeX in more detail.

\section{Pin-Hole soft X-ray Imager-spectrophotometer - PHI}

In order to increase the signal-to-noise ratio, the polarimeter unit will be equipped with a $2 \mathrm{D}$ collimator which will limit the field of view centered on the flare to $\sim 2$ arcmin. Therefore, a reliable pointing system is required as well as a system to localize the source within the coordinate system of KORTES. A simple pin-hole camera will be sufficient for this purpose. In order to achieve adequate X-ray photon statistics, the pin hole will have $\sim 1 \mathrm{~mm}^{2}$ size, a focal length of $60 \mathrm{~cm}$ and will be equipped with a $256 \times 1024$ pixel detector, with a pixel size of $26 \mu \mathrm{m}$ (e2v CCD30-11 Back Illuminated). Such a configuration will project an X-ray solar image of $\sim 5.5 \mathrm{~mm}$, a diameter that corresponds to $\sim 215$ detector pixels. The simulated solar C5.0-class flare image to be seen by this camera is shown in 

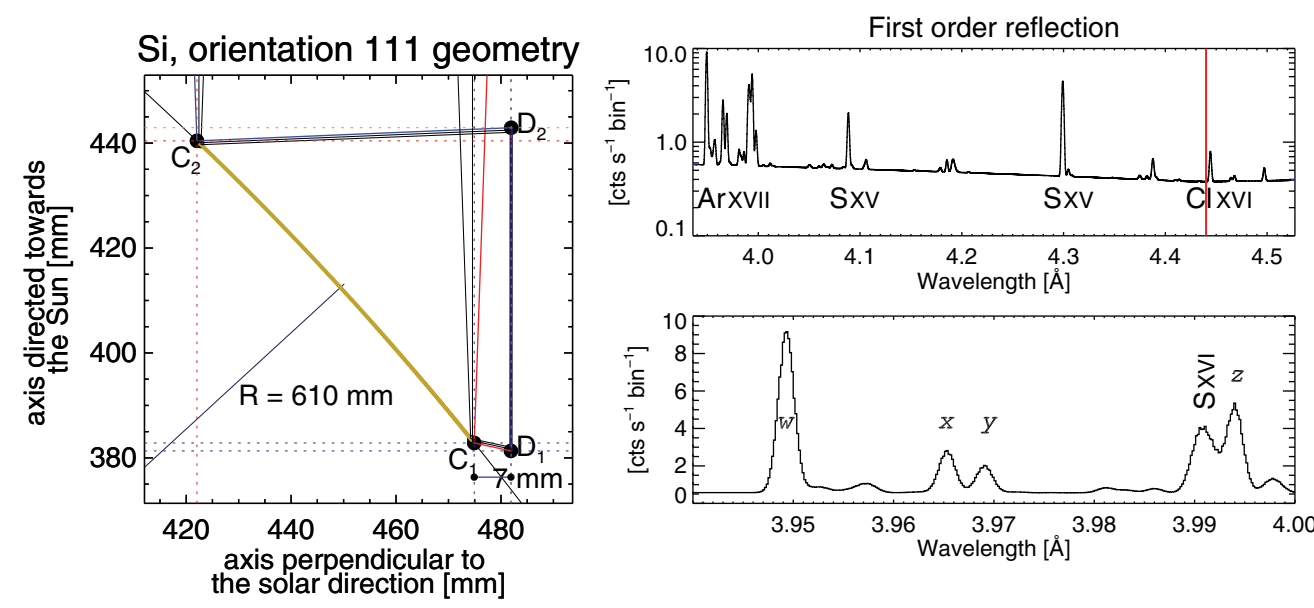

Figure 3. Left: Cylindrical mono-crystal wafer of Si with a $610.0 \mathrm{~mm}$ curvature radius. Points $\mathrm{C}_{1}$ and $\mathrm{C}_{2}$ define the "active" edges of the $\mathrm{Si}$ crystal and $\mathrm{D}_{1}$ and $\mathrm{D}_{2}$ the corresponding edges of the CCD detector. The yellow-green line represents the curved surface of the monocrystal wafer cut along the 111 plane. Blue and red lines show the photon paths for the sources located at the boundary of the 2D collimated FOV (angles are exaggerated for clarity). Right: Simulated spectrum of an M5 flare to be recorded over the entire spectral range (upper-part). The position of the wavelength corresponding to the Brewster angle is indicated with red vertical line. An enlarged portion of the spectrum covering the principal Ar XVII triplet lines is shown below. See the on-line edition of this book for a color version of this figure.

Fig. 2. A large part of the CCD signal from the area outside of the solar disk will be used to determine the particle flux at the ISS during the orbit with high temporal resolution. This signal will also be used to estimate the background for the large polarimeter CCD (see below).

From images similar to that shown in Fig. 2 (to be collected every $0.1 s$ ), the soft Xray light curves of individual active regions will be analyzed in real-time by the on-board software in order to detect and locate flares. A 2D Gaussian elliptical profile will be fitted to individual stronger brightenings and their central position will be determined. This position will be passed to the B-POL pointing system in order to lock the position of the polarimeter's rotation axis on this target. The limb-brightened rim will be used to determine the position of the edge of the solar disk. For low intensity areas, where single counts (per CCD dump) are observed in some pixels, the measurements will be done with moderate spectral resolution $(\sim 200 \mathrm{eV})$.

\section{X-ray polarimetry of solar flares - B-POL}

The heart of B-POL (cf. Fig. 1) consists of a cylindrically bent mono-crystal and a large detector (e2v back illuminated CCD261-84, $2048 \times 4096 ; 30.7 \mathrm{~mm} \times 61.6 \mathrm{~mm}$ ), fixed together at an angle of $\sim 45^{\circ}$. This unit will be continuously rotating along the axis directed to the source selected from the pin-hole image. The rotation rate has been set to $1 s^{-1}$, which was chosen as a compromise between the CCD read-out time and the expected rate of polarization variation (of the order of seconds). The pointing of the rotation axis will usually be moved towards the brightest region of interest (i.e. the flare) by turning the support of the crystal-detector section within the angular range of $\pm 2^{\circ}$. We decided to use a Silicon 111 crystal surface bent cylindrically to a $610.00 \mathrm{~mm}$ radius as the dispersive medium. The adopted crystal-detector geometry setup allows spectra to be obtained in the spectral range $3.9 \AA-4.5 \AA$ with exceptionally good spectral resolution 
Table 1. Crystals selected for the RDS.

\begin{tabular}{lccccc}
\hline No & Crystal & Orientation & $2 \mathrm{~d}[\AA]$ & Wavelenght range $1[\AA]$ & Wavelenght range $2[\AA]$ \\
\hline 1. & $\mathrm{Si}$ & 400 & 2.715 & $1.397-2.331$ & $0.270-1.796$ \\
2. & $\mathrm{Si}$ & 220 & 3.840 & $1.977-3.298$ & $0.391-2.541$ \\
3. & $2 \times \mathrm{Si}^{*}$ & 111 & 6.271 & $3.228-5.385$ & $0.639-4.150$ \\
4. & Quartz & $10 \overline{1} 1$ & 6.684 & $3.441-5.740$ & $0.681-4.423$ \\
5. & Quartz & $10 \overline{1} 0$ & 8.514 & $4.383-7.312$ & $0.868-5.635$ \\
6. & $\mathrm{ADP}$ & 101 & 10.648 & $5.482-9.145$ & $1.086-7.047$ \\
7. & KAP & 001 & 26.640 & $13.72-22.88$ & $12.72-17.63$ \\
\hline
\end{tabular}

( $\sim 0.00014 \AA /$ bin). This is better than the instrumental spectral resolution $(0.00034 \AA)$ and is much smaller than the thermal/turbulent width of the emission lines. Details of the crystal-detector placement and an example of the spectrum to be measured for an M5.0 flare are given in Fig. 3

As shown in Fig. 3, in the selected spectral range a number of strong emission lines are present, due to Ar XVII, Si XV, Si XVI as well as the much weaker Cl XVI triplet lines. The observed spectral range will be recorded for Bragg incidence angles close to the Brewster angle, and so their reflected intensities will depend on the degree of linear polarization and respective orientation of the polarization vector relative to the crystal's surface.

During the rotation of the crystal-detector unit, the position of the crystal dispersion plane with respect to the incoming light beam will continuously change. This will give rise to a modulation of the reflected beam intensity, provided the incident X-ray beam is linearly polarized. It has been shown with the Electron Beam Ion Trap (EBIT: Beiersdorfer et al. 1996) that the polarization of the He-like triplet lines for iron and lighter elements can be substantial and it depends on the relative orientation of the EBIT particle beam and the crystal polarimeter plane. From Fig. 1 of this Paper it follows that, for $\mathrm{Ar}$ XVII (as well as for the much weaker $\mathrm{Cl}$ XVI triplet lines), the degree of polarization of $w$ and $x$ ( see Fig. 3, lower left panel) lines can be close to 0.6 and -0.6 respectively. This level of polarization would easily be detected by B-POL for stronger flares.

The Ar as well as S lines are nearly always present in solar X-ray spectra for activity above GOES B5.0 class (Sylwester et al. 2010). Therefore, the B-POL will record spectra of flares and stronger active regions for most of the time. This will also allow for studies of thermal plasma properties like turbulence, directed motions, differential emission measure distribution and elemental composition. However, lines of only a few elements are present in the B-POL spectral range suitable for polarization detections. In order to extend the spectral coverage over the entire soft X-ray range from $1 \AA$ to $23 \AA$, we included in KORTES the novel RDS spectrometer described below.

\section{The fast-rotating X-ray spectrometer - RDS}

The fast-Rotating Drum X-ray Spectrometer will allow us to investigate very fast changes of the solar spectra, including lines and continuum. Precise line Doppler shift measurements will be possible. The novelty of the approach is that in order to assign a wavelength to each photon, which is Bragg-reflected from the crystal, we will use the timing information on the photon arrival on the SDD detector. Using such modern detectors (Ketek, Vitus-H80), the arrival time can be determined with an accuracy of $\sim 1$ microsecond. In one microsecond, the drum with the crystals will rotate by $\sim 10$ arcsec only, so the line wavelength smear corresponding to such a small change of the incidence angle can be neglected and the actual photon wavelength can be derived with precision better than the width of the instrumental function (i.e. the crystal rocking curve). As seen in Fig. 1 (right panel), the flat crystals are attached to an octogonal drum rotating at a 
high rate $(10 \mathrm{rps})$. The drum geometry is selected such that the solar X-rays illuminate a pair of the rotating crystals, from which the Bragg-reflected photons are being recorded by two pairs of SDD detectors. Each detector records spectra at a slightly different Bragg angle. We will use detectors characterized by a very fast response $(\sim 1 \mu s)$. By measuring the photon arrival times on every detector, the respective crystal incidence angles can easily be determined and converted to the corresponding incoming photon wavelengths (or, equivalently, energies) provided the calibration of the system is carefully done on the ground. A histogram of spectra will be built as time progresses, with the time resolution depending on the strength of the intensity source. For flares of class M5.0 and above, enough photons will be recorded to study spectra variability on a time scale of $\sim 1$ second.

The symmetrical location of detectors on both sides of the drum (with respect to the direction towards the Sun) represents the configuration necessary to fulfill the tasks of a "doppler-meter" (Sylwester et al. 2015). We plan to use eight flat crystals, two of them identical (see Table 1 for details). For every crystal two spectral wavebands are given. The first band is covered by the "front" pair of detectors (those closer to the Sun). The second waveband is covered by the rear pair. Two identical crystals marked with a star are placed in the classical doppler-meter configuration. The combination of all the rotating crystals will enable a continuous spectral range coverage, extending from $0.3 \AA$ to $22.8 \AA$, recorded every $0.1 \mathrm{~s}$. This will allow us to determine spectral line fluxes and and doppler shifts for all abundant elements, from oxygen to iron, emitted by a hot flaring plasma between $1 \mathrm{MK}$ and $50 \mathrm{MK}$. These data will support the measurements of spectra made using the B-POL unit.

\section{Summary}

The SolpeX spectrometer will be placed on the ISS as a part of KORTES Russian instrument. SolpeX consists of a pin-hole imager, the B-POL Bragg polarimeter and the RDS rotating spectrometer with eight flat crystals. With these three units, the following measurements will be performed in the soft X-ray range for flares and/or active regions (AR) of temperatures of $1 \mathrm{MK}$ and above

- individual AR/flare light curves and their coordinates with a time resolution of $0.1 \mathrm{~s}$,

- flare linear polarization vector characteristics at energies around $\sim 3 \mathrm{keV}$ every 1 $10 \mathrm{~s}$,

- AR/flare spectra in the entire soft X-ray range, with a temporal resolution of $0.1 s$.

These measurements will complement each other allowing substantial progress in understanding the processes of magnetic energy release, transport and dissipation in various solar coronal structures, flares in particular. The success of this project will pave the way for building a larger instrument, based on similar principles, to be placed on a larger future solar satellite.

\section{Acknowledgements}

We would like to thank Professor Ken J. H. Phillips for corrections and helpful comments. We acknowledge financial support from the Polish National Science Centre grant 2013/11/B/ST9/00234.

\section{References}

Beiersdorfer, P., Vogel, D. A., Reed, K. J., Decaux, V., Scofield, J. H., Widmann, K., H'olzer, G., F'rster, E., Wehrhan, O., Savin, D. W., \& Schweikhard, L. 1996, Physical Review A 53, 3974

Boggs, S. E., Coburn, W., \& Kalemci, E. 2006, ApJ 638, 1129 
Elwert, G. \& Haug, E. 1970, Solar Phys. 15, 234

Emslie, A. G., Bradsher, H. L., \& McConnell, M. L. 2008, ApJ 674, 570

Emslie, A. G. \& Brown, J. C. 1980, ApJ 237, 1015

Jeffrey, N. L. S. \& Kontar, E. P. 2011, A\&A 536, 93

McConnell, M. L., Smith, D. M., Emslie, G., Lin, R. P., \& Ryan, J. M. 2003, Hard x-ray polarimetry with the Ramaty high energy solar spectroscopic imager (RHESSI) Proc. SPIE 4843,8

Suarez-Garcia, E., Hajdas, W., Wigger, C., Arzner, K., G'del, M., Zehnder, A., \& Grigis, P. 2006, Solar Phys. 239, 149

Sylwester, J., Kordylewski, Z., Płocieniak, S., Siarkowski, M., Kowalinski, M., Nowak, S., Trzebinski, W., Steslicki, M., Sylwester, B., Stanczyk, E., and 5 coauthors 2015, Solar Phys. 290,2

Sylwester, B., Sylwester, J., \& Phillips, K. 2010, A\& $A$ 514, A82

Zharkova, V. V., Kuznetsov, A. A., \& Siversky, T. V. 2010, A $\& A$ 512, 8

Zhitnik, I. A., Logachev, Yu. I., Bogomolov, A. V., Denisov, Yu. I., Kavanosyan, S. S., Kuznetsov, S. N., Morozov, O. V., Myagkova, I. N., Svertilov, S. I., Ignat'ev, A. P., and 3 coauthors 2006, Solar System Research 40, 93 